\title{
On the closure property of Lepage equivalents of Lagrangians
}

\author{
Nicoleta VOICU, Stefan GAROIU, Bianca VASIAN \\ Transilvania University of Brasov, Romania
}

\begin{abstract}
Lepage equivalents of Lagrangians are a higher order, field-theoretical generalization of the notion of Poincaré-Cartan form from mechanics and play a similar role: they give rise to a geometric formulation (and to a geometric understanding) of the calculus of variations.

A long-standing open problem is the determination, for field-theoretical Lagrangians $\lambda$ of order greater than one, of a Lepage equivalent $\Phi_{\lambda}$ with the so-called closure property: $\Phi_{\lambda}$ is a closed differential form if and only if $\lambda$ has vanishing Euler-Lagrange expressions.

The present paper proposes a solution to this problem, for general Lagrangians of order $r \geq 1$. The construction is a local one; yet, we show that in most of the cases of interest for physical applications, the obtained Lepage equivalent $\Phi_{\lambda}$ is actually globally defined.

A variant of this construction, which is convenient in the cases when $\lambda$ is a reducible Lagrangian, is also introduced. In particular, for reducible Lagrangians of order two, the obtained Lepage equivalents are of order one.
\end{abstract}

Corresponding author's e-mail: nico.voicu@unitbv.ro

Keywords: jet bundle, Poincaré-Cartan form, Lepage equivalent of a Lagrangian, VainbergTonti Lagrangian

MSC2020: 58A10, 58A20, 83D05

\section{Introduction}

In classical mechanics, the Poincaré-Cartan form

$$
\Theta_{L}=L d t+\frac{\partial L}{\partial \dot{q}^{\sigma}}\left(d q^{\sigma}-\dot{q}^{\sigma} d t\right)
$$

associated to a Lagrangian $L=L\left(t, q^{\sigma}, \dot{q}^{\sigma}\right)$ is a fundamental concept, giving rise to a geometric understanding of the calculus of variations. Similarly, its higher-order, field theoretical analogue, mainly known under the name of Lepage equivalent of a Lagrangian [10, [13, [5], allows for a concise and elegant description of the apparatus of variational calculus, solely in terms of operations with differential forms. In view of such a description, the Lagrangian itself will be regarded as a differential form $\lambda$ on a certain jet bundle, rather than as a function (for instance, in the above example, $\lambda=L d t)$.

But, whereas in mechanics, the Poincaré-Cartan form is unique, in field theory, any given Lagrangian $\lambda$ admits multiple Lepage equivalents $\theta_{\lambda}$, exhibiting different features. One of the most desirable such features is the so-called closure property:

$$
\lambda \text { is variationally trivial } \Leftrightarrow d \theta_{\lambda}=0 .
$$


Once the closure property is satisfied, all Lagrangians producing the same Euler-Lagrange equations will be characterized by one and the same $d \theta_{\lambda}$, in other words, $d \theta_{\lambda}$ will capture a specific dynamics, not just a particular Lagrangian 1 .

This property was initially motivated by the study of symmetries of the Euler-Lagrange form, see [1, 2], but it is a very promising one in at least two other directions:

- Geometric formulation of Hamiltonian field theory: given a Lagrangian form $\lambda$, a Hamiltonian form $H_{\lambda}$ is constructed via the exterior derivative $d \theta_{\lambda}$ - and generally, it is not guaranteed that Lagrangians that produce the same Euler-Lagrange equations will also lead to the same Hamilton equations. This drawback is eliminated if the mapping $\lambda \mapsto \theta_{\lambda}$ is $\mathbb{R}$-linear and satisfies the closure property.

- Variational sequences (e.g., 9]), where it offers an elegant characterization of the kernel of the Euler-Lagrange mapping.

The closure property is notoriously obeyed in mechanics by the Poincaré-Cartan form $\Theta_{L}$, both in the first order case (11) and for higher order Lagrangians. But, in field theory, finding Lepage equivalents with the closure property has been for many years an open problem. Actually, to the best of our knowledge, mappings $\lambda \mapsto \theta_{\lambda}$ obeying it are only known in some very specific situations:

1. First order Lagrangians. In this case, a globally defined Lepage equivalent with the desired feature, called the fundamental Lepage equivalent $\rho_{\lambda}$, was introduced by Krupka, [11] and rediscovered by Bethounes, 1]; for first order homogeneous Lagrangians, a similar notion was introduced by Urban and Brajercik, 21.

2. Homogeneous Lagrangians with two independent variables; in this case, an extension of the fundamental form $\rho_{\lambda}$ was constructed by Saunders and Crampin, 20].

In the present paper, we propose a general procedure which solves this problem (at least, locally) for general Lagrangians $\lambda$, of any order $r \geq 1$. Our construction relies on a different idea than the first order construction in [11, as it uses as a raw material, another Lepage equivalent, called the principal Lepage equivalent, [10] - which is much simpler; more specifically, it is 1-contact, whereas the fundamental Lepage equivalent $\rho_{\lambda}$ has a higher degree of contactness.

The principal Lepage equivalent, as it stands, does not obey the closure property, but we show that it can be tailored in such a way as to eliminate this drawback, as follows. To any Lagrangian $\lambda$ over a given fibered chart domain, one can canonically attach, on the respective chart domain, a dynamically equivalent Lagrangian: the so-called Vainberg-Tonti Lagrangian $\lambda_{V T}$ of the EulerLagrange form of $\lambda$. The difference between $\lambda$ and $\lambda_{V T}$ is thus a trivial Lagrangian, which can be written, 9], up to pullback by the corresponding jet projections, as

$$
\lambda=\lambda_{V T}+h d \alpha,
$$

where $h$ denotes the horizontalization operator and $d \alpha$ is uniquely determined, via a specific homotopy operator.

Using the above decomposition, we define

$$
\Phi_{\lambda}:=\Theta_{\lambda_{V T}}+d \alpha,
$$

where $\Theta_{\lambda_{V T}}$ is the principal Lepage equivalent of $\lambda_{V T}$ and the equality should be, again, understood up to pullback by some jet projections. The differential form $\Phi_{\lambda}$ is a Lepage equivalent of $\lambda$, which we

\footnotetext{
${ }^{1}$ Moreover, it can be reasonably argued, 7], [13, that, in a lot of considerations (especially, having in view a Hamiltonian picture), it is the exterior derivative $d \theta_{\lambda}$, rather than $\theta_{\lambda}$ itself, that plays the key role.
} 
call canonical. As for variationally trivial Lagrangians $\lambda$, the associated Vainberg-Tonti Lagrangian $\lambda_{V T}$ identically vanishes, the above recipe guarantees that, in this case, $\Phi_{\lambda}=d \alpha$, hence it is closed. Moreover, $\Phi_{\lambda}$ is 1-contact.

As, typically, both the Vainberg-Tonti Lagrangian and the principal Lepage equivalent are only defined over a coordinate chart, a natural question is whether (or, rather, when) is $\Phi_{\lambda}$ globally defined. A complete answer to this question is still to be investigated; yet, we show that, for globally defined Lagrangians of order $r \leq 2$ on tensor bundles, having second order Euler-Lagrange equations - which represent most of the cases of interest for physical theories $-\Phi_{\lambda}$ is actually globally well defined.

A variant of the above construction, which is convenient in the case when $\lambda$ is locally equivalent to a lower order Lagrangian $\lambda^{\prime}$, is to consider in (3), instead of the Vainberg-Tonti Lagrangian $\lambda_{V T}$, a reduced Lagrangian $\lambda^{\prime}$. This leads to (generally, non-unique) Lepage equivalents $\phi_{\lambda}$, which we will call reduced; and, if we can ensure that $\lambda^{\prime}$ is truly of minimal order, the obtained reduced Lepage equivalent will still possess the closure property. In particular, for reducible second order Lagrangians, any reduced Lepage equivalent will be of order 1.

The article is structured as follows. Section 2 is a somewhat didactic presentation of the known results and notions to be used in the following. In Sections 3 and 4, we introduce the canonical, respectively, minimal Lepage equivalents. Section 5 is devoted to examples and Section 6 , to conclusions and future work perspectives.

\section{Preliminaries}

In the following, we present the technical ingredients to be used in our construction. The notions and results presented in this section can be found in more detail, e.g., in the book by Krupka, 9].

In the approach we present below, a Lagrangian is regarded as a differential form on a certain jet bundle of a fibered manifold $(Y, \pi, X)$; in physics, the total space $Y$ of the respective fibered manifold is interpreted as the configuration space of a given system, the base manifold $X$ is typically interpreted as space (or spacetime) and sections of $\pi$ are interpreted as fields. The case $\operatorname{dim} X=1$ corresponds to mechanics and, in this case, sections of $Y$ are curves.

\subsection{Lagrangians, Lepage equivalents and first variation formula}

A fibered manifold is a triple $(Y, \pi, X)$, where $X, Y$ are smooth manifolds, with $\operatorname{dim} X=n$, $\operatorname{dim} Y=m+n$, and $\pi: Y \rightarrow X$ is a surjective submersion; the base manifold $X$ is assumed to be orientable. On a fibered manifold, there exists an atlas consisting of fibered charts $(V, \psi)$, $\psi=\left(x^{i}, y^{\sigma}\right)$, such that $\pi$ is represented in coordinates as $\pi:\left(x^{i}, y^{\sigma}\right) \mapsto\left(x^{i}\right)$. We will denote by $\Gamma(Y)$ the set of local sections $\gamma: U \rightarrow Y$ (where $U \subset X$ is open); elements $\gamma \in \Gamma(Y)$ are represented in a fibered chart as $\gamma:\left(x^{i}\right) \mapsto\left(x^{i}, y^{\sigma}\left(x^{i}\right)\right)$.

Each fibered chart $(V, \psi)$ induces a fibered chart $\left(V^{r}, \psi^{r}\right), \psi^{r}=\left(x^{i}, y^{\sigma}, y_{j_{1}}^{\sigma}, \ldots, y_{j_{1} j_{2} \ldots j_{r}}\right)$ on the $r$-jet prolongation $J^{r} Y$. In these charts, the canonical projections $\pi^{r, s}: J^{r} Y \rightarrow J^{s} Y, J_{x}^{r} \gamma \mapsto J_{x}^{s} \gamma$ $\left(r>s, J^{0} Y:=Y\right)$ are represented as: $\left(x^{i}, y^{\sigma}, y_{j_{1}}^{\sigma}, \ldots, y_{j_{1} j_{2} \ldots j_{r}}^{\sigma}\right) \mapsto\left(x^{i}, y^{\sigma}, y_{j_{1}}^{\sigma}, \ldots, y_{j_{1} j_{2} \ldots j_{s}}^{\sigma}\right)$ and the projection $\pi^{r}: J^{r} Y \rightarrow X, J_{x}^{r} \gamma \mapsto x$, as: $\left(x^{i}, y^{\sigma}, y_{j_{1}}^{\sigma}, \ldots, y_{j_{1} j_{2} \ldots j_{r}}^{\sigma}\right) \mapsto\left(x^{i}\right)$. By $\Omega_{k}\left(W^{r}\right)$ and $\Omega\left(W^{r}\right)$ we will denote the set of $k$-forms (respectively, of all differential forms) defined on $W^{r}:=$ $J^{r} W \subset J^{r} Y$, where $W \subset Y$ is open.

Also, for the simplicity of writing, if there is no risk of confusion, we will sometimes identify forms $\rho$ with their pullbacks $\left(\pi^{s, r}\right)^{*} \rho, s \geq r$; that is, instead of $\left(\pi^{s, r}\right)^{*} \rho=\theta$, we may simply write 
$\rho=\theta$.

Horizontal forms and contact forms on $J^{r} Y$. A differential form $\rho \in \Omega_{k}\left(W^{r}\right)$ is called $\pi^{r}$-horizontal, if $\rho\left(\Xi_{1}, \ldots, \Xi_{k}\right)=0$ whenever one of the vector fields $\Xi_{i}, i=\overline{1, k}$, is $\pi^{r}$-vertical (i.e., $T \pi^{r}\left(\Xi_{i}\right)=0$ ); in a fibered chart, any horizontal form on $\Omega_{k}\left(W^{r}\right)$ is expressed as a linear combination of the wedge products $d x^{i_{1}} \wedge d x^{i_{2}} \wedge \ldots \wedge d x^{i_{k}}$. Yet, for a more compact writing, it is advantageous to use the following locally defined forms:

$$
\begin{aligned}
& \omega_{0}:=d x^{1} \wedge \ldots \wedge d x^{n}, \quad \omega_{i}:=\mathbf{i}_{\partial_{i}} \omega_{0}=(-1)^{i-1} d x^{1} \wedge \ldots \wedge \widehat{d x^{i} \wedge \ldots \wedge d x^{n},} \\
& \omega_{i_{1} \ldots i_{k}}:=\mathbf{i}_{\partial_{i_{k}}} \mathbf{i}_{\partial_{i_{k-1}}} \ldots \mathbf{i}_{\partial_{i_{1}}} \omega_{0},
\end{aligned}
$$

where $\mathbf{i}$ denotes interior product. This way, any $\pi^{r}$-horizontal $k$-form on $W^{r}(k \leq n)$ will have a coordinate expression:

$$
\rho=\frac{1}{k !} A^{i_{1} \ldots i_{k}} \omega_{i_{1} \ldots i_{k}}
$$

where $A^{i_{1} \ldots i_{k}}$ are smooth functions of $x^{i}, y^{\sigma}, y_{j_{1}}^{\sigma}, \ldots, y_{j_{1} j_{2} \ldots j_{r}}^{\sigma}$. The set of $\pi^{r}$-horizontal $k$-forms over $W$ will be denoted by $\Omega_{k, X}\left(W^{r}\right)$.

Similarly, one can define $\pi^{r, s}$-horizontal forms, $0 \leq s \leq r$; locally, these are generated by wedge products of $d x^{i}, d y^{\sigma}, \ldots, d y_{j_{1} \ldots j_{s}}^{\sigma}$.

A form $\theta \in \Omega\left(W^{r}\right)$ is called a contact form if it vanishes along all prolonged sections, i.e., $J^{r} \gamma^{*} \theta=0, \forall \gamma \in \Gamma(Y)$. For instance:

$$
\begin{aligned}
\omega^{\sigma} & =d y^{\sigma}-y_{j}^{\sigma} d x^{j} \\
\omega_{i_{1} i_{2} \ldots i_{k}}^{\sigma} & =d y_{i_{1} i_{2} \ldots i_{k}}^{\sigma}-y_{i_{1} i_{2} \ldots i_{k} j}^{\sigma} d x^{j}
\end{aligned}
$$

where $k \leq r-1$, represent contact forms; more precisely, they are elements of a local basis of $\Omega_{1}\left(W^{r}\right)$, called the contact basis: $\left\{d x^{i}, \omega^{\sigma}, \ldots, \omega_{i_{1} \ldots i_{r-1}}^{\sigma}, d y_{i_{1} \ldots i_{r}}^{\sigma}\right\}$.

A $k$-form $\theta \in \Omega_{k}\left(W^{r}\right)$ is called $l$-contact $(l \leq k)$ if $\mathbf{i}_{\Xi_{1}} \mathbf{i}_{\Xi_{2}} \ldots \mathbf{i}_{\Xi_{l}} \theta$ is horizontal whenever the vector fields $\Xi_{1}, \ldots, \Xi_{l}$ are all $\pi^{r}$-vertical; in the contact basis, in the expression of an $l$-contact $k$-form, each term contains precisely $l$ of the contact 1-forms (77)-(8) (and $k-l$ of the forms $d x^{i}$ ).

Rising to $J^{r+1} Y$, any $\rho \in \Omega_{k}\left(W^{r}\right)$ can be uniquely split into a $\pi^{r}$-horizontal part $h \rho \in \Omega_{k}\left(W^{r+1}\right)$ and a contact part $p \rho \in \Omega_{k}\left(W^{r+1}\right)$ :

$$
\left(\pi^{r+1, r}\right)^{*} \rho=h \rho+p \rho
$$

the contact part can be in its turn decomposed as

$$
p \rho=p_{1} \rho+\ldots+p_{k} \rho,
$$

where the form $p_{l} \rho$ is $l$-contact, $l=1, \ldots, k$. On the other hand, the mapping $\Omega\left(W^{r}\right) \rightarrow$ $\Omega\left(W^{r+1}\right), \rho \mapsto h \rho$ in (9), is a morphism of exterior algebras, called horizontalization; it acts on functions $f: J^{r} Y \rightarrow \mathbb{R}$ as:

$$
h f=f \circ \pi^{r+1, r}, \quad h d f=d_{i} f d x^{i},
$$

where $d_{i}$ denotes total $x^{i}$-derivative (of order $r+1$ ): $d_{i} f:=\partial_{i} f+\frac{\partial f}{\partial y^{\sigma}} y_{i}^{\sigma}+\ldots \frac{\partial f}{\partial y_{j_{1} \ldots j_{r}}^{\sigma}} y_{j_{1} \ldots j_{r} i}^{\sigma}$. For instance, $h d x^{i}=d x^{i}, \quad h d y^{\sigma}=y_{i}^{\sigma} d x^{i}, \ldots, h d y_{j_{1} \ldots j_{r}}^{\sigma}=y_{j_{1} \ldots j_{r} i}^{\sigma} d x^{i}$. 
The horizontal part $h \rho$ is the only one that survives of $\rho$ when pulled back by prolonged sections $J^{r} \gamma$; more precisely, for any $\gamma \in \Gamma(Y): J^{r} \gamma^{*} \rho=J^{r+1} \gamma^{*}(h \rho)$.

Here are two important classes of differential forms on $W^{r} \subset J^{r} Y$ :

1. Lagrangians: A Lagrangian of order $r$ is, by definition, a $\pi^{r}$-horizontal form $\lambda \in \Omega_{n, X}\left(W^{r}\right)$ of rank $n=\operatorname{dim} X$; in fibered coordinates, a Lagrangian is expressed as:

$$
\lambda=\mathcal{L} \omega_{0}, \quad \mathcal{L}=\mathcal{L}\left(x^{i}, y^{\sigma}, \ldots, y_{i_{1} \ldots i_{r}}^{\sigma}\right)
$$

where $\omega_{0}$ is as in (4).

2. Source forms (or dynamical forms, [18]) are defined as $\pi^{r, 0}$-horizontal, 1-contact $(n+1)$ forms; in fibered coordinates, any source form $\varepsilon \in \Omega_{n+1}\left(W^{r}\right)$ is represented as:

$$
\varepsilon=\varepsilon_{\sigma} \omega^{\sigma} \wedge \omega_{0}, \quad \varepsilon_{\sigma}=\varepsilon_{\sigma}\left(x^{i}, y^{\sigma}, \ldots, y_{i_{1} \ldots i_{r}}^{\sigma}\right),
$$

where $\omega^{\sigma}$ are as in (7). The most prominent particular case of source forms are Euler-Lagrange forms of Lagrangians, to be discussed below.

Lepage equivalents and first variation formula. Consider a Lagrangian $\lambda \in \Omega_{n, X}\left(W^{r}\right)$ of order $r$. The action attached to $\lambda$ and to a compact domain $D \subset X$ is the function $S: \Gamma(Y) \rightarrow \mathbb{R}$, given by:

$$
S(\gamma)=\int_{D} J^{r} \gamma^{*} \lambda
$$

The variation of $S$ under the flow of a $\pi$-projectable vector field $\Xi=\xi^{i} \partial_{i}+\Xi^{\sigma} \partial_{\sigma}$ on $Y$ is then:

$$
\delta_{\Xi} S(\gamma)=\int_{D} J^{r} \gamma^{*} \mathfrak{L}_{J^{r} \Xi \lambda,}
$$

where the symbol $\mathfrak{L}$ stands for Lie derivative.

A Lepage equivalent of a Lagrangian $22 \in \Omega_{n, X}\left(W^{r}\right)$ is an $n$-form $\theta_{\lambda} \in \Omega_{n}\left(W^{s}\right)$ on some jet prolongation $J^{s} Y$, with the following properties:

(1) $\theta_{\lambda}$ and $\lambda$ define the same variational problem, i.e., up to the corresponding jet projections:

$$
h \theta_{\lambda}=\lambda .
$$

(2) $\mathcal{E}_{\lambda}:=p_{1} d \theta_{\lambda}$ is a source form.

Condition (1) should be understood as follows. For any section $\gamma \in \Gamma(Y)$, there holds: $J^{s} \gamma^{*} \theta_{\lambda}=$ $J^{r} \gamma^{*} \lambda$, which means that we can substitute $J^{s} \gamma^{*} \theta_{\lambda}$ for $J^{r} \gamma^{*} \lambda$ into the action (11). Condition (2) actually states that $p_{1} d \theta_{\lambda}$ must be locally generated by $\omega^{\sigma}$ and $d x^{i}$ alone (no higher order elements $\omega^{\sigma}{ }_{i_{1} \ldots i_{k}}$ of the contact basis).

\footnotetext{
${ }^{2} \mathrm{~A}$ related, but distinct, notion is the one of Lepage equivalent of a source form $\varepsilon$, 18. In the following, whenever we refer to Lepage equivalents, we will automatically mean Lepage equivalents of Lagrangians.
} 
Given any Lepage equivalent $\theta_{\lambda} \in \Omega_{n}\left(W^{s}\right)$ of $\lambda$, Cartan's formula $\mathfrak{L}_{J^{r} \Xi} \theta_{\lambda}=d \mathbf{i}_{J^{r} \Xi} \theta_{\lambda}+\mathbf{i}_{J^{r} \Xi} d \theta_{\lambda}$ applied to the integrand in (12) yields:

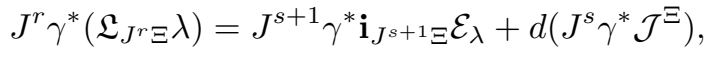

where:

(i) the source form $\mathcal{E}_{\lambda}=p_{1} d \theta_{\lambda} \in \Omega_{n}\left(W^{s+1}\right)$ (of order $s+1 \leq 2 r$ ) is the Euler-Lagrange form of $\lambda$, locally given by

$$
\begin{aligned}
\mathcal{E}_{\lambda} & =\mathcal{E}_{\sigma}(\lambda) \omega^{\sigma} \wedge \omega_{0}, \\
\mathcal{E}_{\sigma}(\lambda) & =\frac{\delta \mathcal{L}}{\delta y^{\sigma}}=\frac{\partial \mathcal{L}}{\partial y^{\sigma}}-d_{i} \frac{\partial \mathcal{L}}{\partial y_{i}^{\sigma}}+\ldots+(-1)^{r} d_{i_{1}} \ldots d_{i_{r}} \frac{\partial \mathcal{L}}{\partial y_{i_{1} \ldots i_{r}}^{\sigma}} .
\end{aligned}
$$

The Euler-Lagrange form $\mathcal{E}_{\lambda}$ does not depend on the choice of the Lepage equivalent $\theta_{\lambda}$.

A local section $\gamma \in \Gamma(Y)$ is critical for the Lagrangian $\lambda$ if and only if, for any $\pi$-vertical vector field $\Xi \in \mathcal{X}(Y)$, there holds:

$$
J^{s+1} \gamma^{*}\left(\mathbf{i}_{J^{s+1}} \Xi \mathcal{E}_{\lambda}\right)=0,
$$

which in coordinates, becomes equivalent to the relations $\mathcal{E}_{\sigma}(\lambda) \circ J^{s+1} \gamma=0$.

(ii) $\mathcal{J}^{\Xi}:=h \mathbf{i}_{J^{s} \Xi} \theta_{\lambda} \in \Omega_{n-1}\left(J^{s+1} Y\right)$ is interpreted as a Noether current.

Every Lagrangian $\lambda \in \Omega_{n, X}\left(W^{r}\right)$ admits Lepage equivalents. The most frequently used one, called the principal Lepage equivalent, is a 1-contact form of order $\leq 2 r-1$ and it is given by:

$$
\Theta_{\lambda}=\mathcal{L} \omega_{0}+\sum_{k=0}^{r-1}\left(\sum_{l=0}^{r-1-k}(-1)^{l} d_{p_{1}} \ldots d_{p_{l}} \frac{\partial \mathcal{L}}{\partial y_{j_{1} \ldots j_{k} p_{1} \ldots p_{l} i}^{\sigma}} \omega_{j_{1} \ldots j_{k}}^{\sigma}\right) \wedge \omega_{i} .
$$

Generally, the principal Lepage equivalent $\Theta_{\lambda}$ is defined only locally; yet, for first and second order Lagrangians, it is globally defined whenever $\lambda$ itself is globally defined, 9], [18].

In particular, for second order Lagrangians $\lambda=\mathcal{L}\left(x^{i}, y^{\sigma}, y_{i}^{\sigma}, y^{\sigma}{ }_{i j}\right) \omega_{0}$, the above formula gives:

$$
\Theta_{\lambda}=\mathcal{L} \omega_{0}+B^{i}{ }_{\sigma} \omega^{\sigma} \wedge \omega_{i}+B_{\sigma}^{i j} \omega_{j}^{\sigma} \wedge \omega_{i}
$$

where:

$$
B_{\sigma}^{i}=\frac{\partial \mathcal{L}}{\partial y^{\sigma}{ }_{i}}-d_{j}\left(\frac{\partial \mathcal{L}}{\partial y_{i j}^{\sigma}}\right), \quad B_{\sigma}^{i j}=\frac{\partial \mathcal{L}}{\partial y^{\sigma}{ }_{i j}} .
$$

Proposition 1 If $\lambda=\mathcal{L} \omega_{0} \in \Omega_{n, X}\left(W^{r}\right)$ is affine in the highest order variables $y_{i_{1} \ldots i_{r}}^{\sigma}$, the order of $\Theta_{\lambda}$ is actually, at most $2 r-2$.

Proof. The statement follows immediately by inspecting the highest order term $d_{p_{1} \ldots} \ldots d_{p_{r-1}} \frac{\partial \mathcal{L}}{\partial y_{p_{1} \ldots p_{r-1} i}^{\sigma}}$ of $\Theta_{\lambda}$.

Any other Lepage equivalent $\theta_{\lambda}$ of $\lambda$ can be locally decomposed as:

$$
\theta_{\lambda}=\Theta_{\lambda}+d \nu+\mu
$$

where $\nu$ is 1-contact and $\mu$ is 2-contact. In particular, any 1-contact Lepage equivalent of $\lambda$ can be expressed, [18, up to the corresponding jet projections, as:

$$
\theta_{\lambda}=\Theta_{\lambda}+p_{1} d \nu .
$$




\subsection{The closure property}

A Lagrangian $\lambda \in \Omega_{n, X}\left(W^{r}\right)$ is called trivial (or null) if its Euler-Lagrange form $\mathcal{E}_{\lambda}$ vanishes identically. It is known, e.g., 9], p. 123, that $\lambda$ is trivial if and only if, for any fibered chart domain $V^{r}=J^{r} V \subset W^{r}$, there exists an $(n-1)$-form $\alpha \in \Omega_{n-1}\left(V^{r-1}\right)$ of order $r-1$, such that:

$$
\lambda=h d \alpha .
$$

A mapping $\theta: \Omega_{n, X}\left(W^{r}\right) \rightarrow \Omega_{n}\left(W^{s}\right)$ attaching to any Lagrangian $\lambda \in \Omega_{n, X}\left(J^{r} Y\right)$, a Lepage equivalent $\theta_{\lambda}$ of some order $s$, is said, [20], to have the closure property, if:

$$
\lambda-\text { trivial } \Rightarrow d \theta_{\lambda}=0,
$$

for all $\theta_{\lambda}$ in the image of $\lambda$.

Remark. The converse implication: $d \theta_{\lambda}=0 \Rightarrow \lambda$ - trivial, is true for any Lepage equivalent $\theta_{\lambda}$, since $d \theta_{\lambda}=0$ implies $\mathcal{E}_{\lambda}=p_{1} d \theta_{\lambda}=0$; hence, whenever it holds, (23) is actually an equivalence.

A first consequence of the closure property is the following.

Proposition 2 : If the mapping $\lambda \mapsto \theta_{\lambda}: \Omega_{n, X}\left(W^{r}\right) \rightarrow \Omega_{n}\left(W^{s}\right)$ is $\mathbb{R}$-linear and has the closure property, then, for any two dynamically equivalent Lagrangians $\lambda, \lambda^{\prime} \in \Omega_{n, X}\left(W^{r}\right)$ :

$$
d \theta_{\lambda}=d \theta_{\lambda^{\prime}} .
$$

Proof. Assuming that the Lagrangians $\lambda, \lambda^{\prime} \in \Omega_{n, X}\left(W^{r}\right)$ are equivalent, it follows that the difference $\lambda-\lambda^{\prime}$ is a trivial Lagrangian, hence $d \theta_{\lambda-\lambda^{\prime}}=0$, which, by linearity, implies (24).

The closure property is a very convenient one for physical applications, as, basically, equality (24) says that all Lagrangians describing the same physics will produce the same $d \theta_{\lambda}$; this can be used, for instance, in:

1. Geometric formulation of Hamiltonian field theory based on Lepage equivalents, see, e.g., 12, 16, 15. Fix, for the moment, an arbitrary Lepage equivalent $\theta_{\lambda} \in \Omega_{n}\left(W^{s}\right)$ of a given Lagrangian $\lambda \in \Omega_{n, X}\left(W^{r}\right)$. A local section $\delta$ of the fibered manifold $\left(J^{s} Y, \pi^{s}, X\right)$ is called a Hamilton extremal of $\theta_{\lambda}$, if, for any $\pi^{s}$-vertical vector field $\xi$ on $J^{s} Y$ :

$$
\delta^{*} \mathbf{i}_{\xi} d \theta_{\lambda}=0
$$

Thus, the Hamilton equation (25) depends not only on $\lambda$, but also on the choice of the Lepage equivalent $\theta_{\lambda}$. In particular, it is not guaranteed that Lagrangians producing the same EulerLagrange equation (16) would generally also produce the same Hamilton equation - which is a major drawback. This drawback can, yet, be eliminated if the (RR-linear) mapping $\lambda \mapsto \theta_{\lambda}$ has the closure property, as, in this case, (24) ensures that, for all equivalent Lagrangians $\lambda, \lambda^{\prime}$, the resulting Hamilton equation (25) will be the same 3 .

\footnotetext{
${ }^{3}$ Of course, the closure property alone does not guarantee that the resulting Hamilton equation, albeit unique, is also equivalent to the Euler-Lagrange equation for $\lambda$ - this will require some extra care in the choice of the Lepage equivalent, as we will see later.
} 
2. Symmetries of the Euler-Lagrange form, [1, 2]. Assuming that a fiber preserving diffeomorphism $\alpha: Y \rightarrow Y$ is a symmetry of the Euler-Lagrange form $\mathcal{E}_{\lambda}=p_{1} d \theta_{\lambda}$, i.e., $J^{s} \alpha^{*} \mathcal{E}_{\lambda}=\mathcal{E}_{\lambda}$ for some given Lagrangian $\lambda \in \Omega_{n, X}\left(W^{r}\right)$, then $\lambda$ and $\lambda^{\prime}:=J^{r} \alpha^{*} \lambda$ must differ by a trivial Lagrangian, which means, by (24) that:

$$
d \theta_{J^{r} \alpha^{*} \lambda}=d \theta_{\lambda} .
$$

If, in addition, $\theta$ obeys the so-called mapping property: $\theta_{J^{r} \alpha^{*} \lambda}=J^{r} \alpha^{*} \theta_{\lambda}$, then, one obtains an even stronger statement: any symmetry of $\lambda$ is a symmetry of $\theta_{\lambda}$ and any symmetry of $\mathcal{E}_{\lambda}$ will also be a symmetry of $d \theta_{\lambda}$ (the converse implications are true for any Lepage equivalent $\left.\theta_{\lambda}\right)$.

Example: The fundamental (Krupka) Lepage equivalent for first order Lagrangians. For $\lambda \in \Omega_{n, X}\left(J^{1} Y\right)$, a globally defined, first order Lepage equivalent possessing the closure (and also, the mapping) property is, [18, [20]:

$$
\rho_{\lambda}=\mathcal{L} \omega_{0}+\sum_{k=1}^{\min \{m, n\}} \frac{1}{(k !)^{2}} \frac{\partial^{k} \mathcal{L}}{\partial y_{i_{1}}^{\sigma_{1}} \ldots \partial y_{i_{k}}^{\sigma_{k}}} \omega^{\sigma_{1}} \wedge \ldots \wedge \omega^{\sigma_{k}} \wedge \omega_{i_{1} \ldots i_{k}} ;
$$

the degree of contactness of $\rho_{\lambda}$ is $\min \{m, n\}$. In the case of second order Lagrangians, an extension of the fundamental Lepage equivalent was recently proposed in 17, but it is not known whether it has the closure property or not.

\subsection{The Vainberg-Tonti Lagrangian of a source form}

Given a source form $\varepsilon$ defined on some fibered chart domain $V^{r} \subset J^{r} Y$, one can canonically attach to $\varepsilon$ and to the respective chart a Lagrangian called the Vainberg-Tonti Lagrangian, with the following property: if the source form $\varepsilon$ admits a Lagrangian on $V^{r}$, then the Vainberg-Tonti Lagrangian is a Lagrangian for $\varepsilon$. The results in this subsection can be found in more detail in Sections 4.9 and 2.7 of 9 .

Consider a fibered chart $(V, \psi)$ on $Y$ and denote $V^{r}:=J^{r} V$. We assume that the image $\psi(V) \subset$ $\mathbb{R}^{m+n}$ is vertically star-shaped, i.e., for any $\left(x^{i}, y^{\mu}\right) \in \psi(V)$, the whole segment $\left(x^{i}, t y^{\mu}\right), t \in[0,1]$ remains in $\psi(V)$. Under this assumption 4 , the correspondence

$$
\chi:\left(t,\left(x^{i}, y^{\sigma}, y_{i}^{\sigma}, \ldots, y_{i_{1} \ldots i_{r}}^{\sigma}\right)\right) \mapsto\left(x^{i}, t y^{\sigma}, t y_{i}^{\sigma}, \ldots, t y_{i_{1} \ldots i_{r}}^{\sigma}\right)
$$

gives rise to a well defined mapping $\chi:[0,1] \times V^{r} \rightarrow V^{r}$. Further, for any $\rho \in \Omega_{k}\left(V^{r}\right)$, set:

$$
I \rho:=\int_{0}^{1} \rho^{(0)}(t) d t
$$

where $\rho^{(0)}(t) \in \Omega_{k-1}\left(V^{r}\right)$ is obtained from the decomposition:

$$
\chi^{*} \rho=d t \wedge \rho^{(0)}(t)+\rho^{\prime}(t)
$$

\footnotetext{
${ }^{4}$ The construction can also be extended to cases when $\psi(V)$ is not vertically star-shaped, see, e.g., 8$]$.
} 
into a $d t$-term and a term $\rho^{\prime}(t)$ which does not contain $d t$. The obtained mapping $I: \Omega_{k}\left(V^{r}\right) \rightarrow$ $\Omega_{k-1}\left(V^{r}\right)$, called the fibered homotopy operator, is $\mathbb{R}$-linear and obeys:

$$
\rho=I d \rho+d I \rho+\left(\pi^{r}\right)^{*} \rho_{0},
$$

where

$$
\rho_{0}:=0^{*} \rho
$$

and 0 denotes the zero section $0:\left(x^{i}\right) \mapsto\left(x^{i}, 0,0, \ldots, 0\right)$ of $V^{r}$. The $k$-form $\rho_{0}$ is defined over $\pi(V) \subset X$.

The following properties will be useful in the following:

$$
I h \rho=0, \quad I p_{k} \rho=p_{k-1} I \rho, \quad 1 \leq k \leq q .
$$

Applying the above operator $I$ to a source form $\varepsilon=\varepsilon_{\sigma} \omega^{\sigma} \wedge \omega_{0} \in \Omega_{n+1}\left(V^{r}\right)$, the obtained $n$-form

$$
\lambda_{\varepsilon}:=I \varepsilon
$$

is a Lagrangian on $V^{r}$, called the Vainberg-Tonti Lagrangian attached to $\varepsilon$. In coordinates:

$$
\lambda_{\varepsilon}=\mathcal{L}_{0} \omega_{0}, \quad \mathcal{L}_{0}=y^{\sigma} \int_{0}^{1} \varepsilon_{\sigma}\left(x^{i}, t y^{\mu}, t y_{i}^{\mu}, \ldots t y_{i_{1} \ldots i_{r}}^{\mu}\right) d t .
$$

If the source form $\varepsilon$ admits a Lagrangian on $V$, then: $\mathcal{E}_{\lambda_{\varepsilon}}=\varepsilon$.

Remark. The Vainberg-Tonti Lagrangian $\lambda_{\varepsilon}$ of a source form $\varepsilon$ is of the same order as $\varepsilon$; in particular, for a second order source form, $\lambda_{\varepsilon}$ is also of second order. That is, very often (e.g., in classical mechanics), $\lambda_{\varepsilon}$ can be order-reduced, i.e., it is equivalent to a lower order Lagrangian.

\section{Canonical Lepage equivalent}

\subsection{Definition and properties}

In the following, for a given Lagrangian $\lambda$ of arbitrary order $r$, we will build a local Lepage equivalent $\Phi_{\lambda}$, possessing the closure property; the obtained Lepage equivalent is 1-contact and of order $4 r-2$.

Fix a fibered coordinate chart $(V, \psi)$ as above and arbitrary Lagrangian $\lambda \in \Omega_{n, X}\left(V^{r}\right)$ of order $r \geq 2$. As, by definition, $\lambda$ is a Lagrangian for its own Euler-Lagrange form $\mathcal{E}_{\lambda}=E_{\sigma} \omega^{\sigma} \wedge \omega_{0}$, the Vainberg-Tonti Lagrangian (of order $\leq 2 r$ )

$$
\lambda_{V T}:=I \mathcal{E}_{\lambda}
$$

is always equivalent to $\lambda$. The difference between $\lambda$ and $\lambda_{V T}$ is thus a trivial Lagrangian; more precisely, one can write, see Lemma 8, Sec. 4.9 of [9]:

$$
\left(\pi^{2 r, r}\right)^{*} \lambda=\lambda_{V T}+h d \alpha
$$


where

$$
\alpha:=I \Theta_{\lambda}+\left(\pi^{2 r-1}\right)^{*} \mu_{0}
$$

and $\mu_{0}$ is an $(n-1)$-form on $\pi(V) \subset X$ such that

$$
0^{*} \Theta_{\lambda}=d \mu_{0}
$$

( $\mu_{0}$ is guaranteed to exist, as $0^{*} \Theta_{\lambda}$ is a form of maximal degree on $X$ ).

We will call the Lagrangian $\lambda_{V T}$, the Vainberg-Tonti Lagrangian associated to $\lambda$.

Let us make the following remarks.

1. As $\Theta_{\lambda}$ is 1-contact, we obtain by (33) that $\alpha$ is horizontal; moreover, since $\Theta_{\lambda}$ is generally of order $2 r-1$, it follows that $\alpha \in \Omega_{n, X}\left(V^{2 r-1}\right)$, i.e., its coordinate expression is:

$$
\alpha=\alpha^{i} \omega_{i}, \quad \alpha^{i}=\alpha^{i}\left(x^{j}, y_{j}^{\sigma}, \ldots, y_{j_{1} \ldots j_{2 r-1}}^{\sigma}\right)
$$

2. For a Lagrangian $\lambda$ of order $r$, the Euler-Lagrange expressions (15) are of order $\leq 2 r$, but their dependence on the variables $y_{i_{1} \ldots i_{2 r}}^{\sigma}$ is, in any fibered chart, at most affine. Hence, the associated Vainberg-Tonti Lagrangian $\lambda_{V T}$ is also at most affine in $y_{i_{1} \ldots i_{2 r}}^{\sigma}$. Consequently, using Proposition 1, we find out that the order of the principal Lepage equivalent $\Theta_{\lambda_{V T}}$ does not exceed $4 r-2$.

We are now able to prove the following result.

Theorem 3 Let $\lambda \in \Omega_{n, X}\left(V^{r}\right)$ be an arbitrary Lagrangian of order $r$, over the vertically star-shaped fibered chart domain $V$ and $\lambda_{V T}=I \mathcal{E}_{\lambda} \in \Omega_{n, X}\left(V^{2 r}\right)$, its associated Vainberg-Tonti Lagrangian. Then:

(i) The differential form $\Phi_{\lambda} \in \Omega_{n}\left(V^{4 r-2}\right)$ given by:

$$
\Phi_{\lambda}:=\Theta_{\lambda_{V T}}+\left(\pi^{4 r-2,2 r-1}\right)^{*} d \alpha,
$$

where $\alpha$ is given by (38)-(39), is a Lepage equivalent of $\lambda$;

(ii) If $\lambda$ is a trivial Lagrangian, then $d \Phi_{\lambda}=0$.

Proof. (i) Write $\lambda$ as in (37). Then, since the horizontalization $h$ is a linear mapping, we have, up to the corresponding jet projections:

$$
h \Phi_{\lambda}=h \Theta_{\lambda_{V T}}+h d \alpha=\lambda_{V T}+h d \alpha=\lambda ;
$$

moreover, taking the exterior derivative of (37), we obtain: $d \Phi_{\lambda}=d \Theta_{\lambda_{V T}}$, therefore,

$$
p_{1} d \Phi_{\lambda}=p_{1} d \Theta_{\lambda_{V T}}=\mathcal{E}_{\lambda_{V T}}=\mathcal{E}_{\lambda},
$$

which proves that $\Phi_{\lambda}$ is a Lepage equivalent of $\lambda$.

(ii) Assuming that $\lambda$ is trivial, we have $\mathcal{E}_{\lambda}=0$, which implies $\lambda_{V T}=0$ and, accordingly, $\Theta_{\lambda_{V T}}=0$; as a consequence, $\Phi_{\lambda}=\left(\pi^{4 r-2,2 r-1}\right)^{*} d \alpha$ is locally exact - therefore, closed.

We will call the differential form $\Phi_{\lambda}$ in (37)-(40), the canonical Lepage equivalent of $\lambda$.

\section{Remarks.}


1. (Uniqueness of $\left.\Phi_{\lambda}\right)$ : Though the $(n-1)$-form $\mu_{0}$ in (39) is not unique, in the expression of $\Phi_{\lambda}$, it only appears through

$$
d \alpha=d I \Theta_{\lambda}+\left(\pi^{2 r-1}\right)^{*} d \mu_{0}=d I \Theta_{\lambda}+\left(\pi^{2 r-1}\right)^{*} 0^{*} \Theta_{\lambda}
$$

which is uniquely defined.

2. Linearity of $\Phi$ : All the mappings $(I, \Theta, \mathcal{E})$ involved in constructing $\Phi$ are $\mathbb{R}$-linear ones, therefore,

$$
\Phi: \Omega_{n, X}\left(V^{r}\right) \mapsto \Omega_{n}\left(V^{4 r-2}\right), \quad \lambda \mapsto \Phi_{\lambda}
$$

is also an $\mathbb{R}$-linear mapping. Together with the closure property, this ensures that, for equivalent Lagrangians $\lambda_{1}, \lambda_{2}$, we will have $d \Phi_{\lambda_{1}}=d \Phi_{\lambda_{2}}$.

Lagrangians admitting globally defined canonical Lepage equivalents. As the above construction heavily relies on quantities that are defined on a specified chart, such as the VainbergTonti Lagrangian and the principal Lepage equivalent, a natural question is whether (and when) could $\Phi_{\lambda}$ be globally defined. Though a complete answer to this question is out of the scope of this paper, here is a result which covers a lot of the situations of interest for physical applications.

Theorem 4 Assume that $Y$ is a tensor bundle over $X$ and $\lambda \in \Omega_{n, X}\left(J^{r} Y\right)$ is a Lagrangian of order at most 2, having second order Lagrange equations. Then, the canonical Lepage equivalent (40) is globally well defined.

Proof. Let us start by the following remark on the fibered homotopy operator $I$ introduced in (29). In the particular case when $(Y, \pi, X)$ has a vector bundle structure, the fiber rescalings $\chi_{t}=\chi(t, \cdot)$, $t \in \mathbb{R}$, are nothing but the jet prolongations of the fiberwise scalar multiplication $v \mapsto t v$ on $Y$, i.e., they make sense globally on $J^{r} Y$. Accordingly, $\chi: \mathbb{R} \times J^{r} Y \rightarrow J^{r} Y,\left(t, J_{x}^{r} \gamma\right) \mapsto \chi_{t}\left(J_{x}^{r} \gamma\right)$ is a well defined, smooth mapping. Hence, for any globally defined form $\rho \in \Omega\left(J^{r} Y\right), \chi^{*} \rho$ is also globally defined on $\mathbb{R} \times J^{r} Y$. Further, noticing that, in (30), we can actually write $\rho^{(0)}=\mathbf{i}_{\partial_{t}}\left(\chi^{*} \rho\right)$, we obtain

$$
I \rho=\int_{0}^{1} \mathbf{i}_{\partial_{t}}\left(\chi^{*} \rho\right) d t,
$$

where, in this case, all the involved operations make sense globally. Therefore, on vector bundles, $I \rho$ is globally defined.

Assume now that $\lambda$ satisfies the above hypotheses; as $\lambda$ is globally defined, its Euler-Lagrange form $\mathcal{E}_{\lambda}$ is also globally defined. Using the above remark, we get that $\lambda_{V T}=I \mathcal{E}_{\lambda}$ is globally defined - and, according to our hypothesis, of second order. But, for second order Lagrangians, the principal Lepage equivalent is globally defined, which means that so is $\Theta_{\lambda_{V T}}$.

On the other hand, as the order of $\lambda$ does not exceed two, $\Theta_{\lambda}$ is globally well defined. Applying again the above remark on the operator $I$, we finally get that $d \alpha=d I \Theta_{\lambda}+\left(\pi^{2 r-1}\right)^{*} 0^{*} \Theta_{\lambda}$, is also globally defined. Summing up, we obtain that both terms of $\Phi_{\lambda}$ are globally defined, which completes the proof.

The above result applies, for instance, to:

- all generally covariant, first order Lagrangians on tensor bundles; 
- Lovelock gravity, Horndeski theories, metric-affine gravity theories with second order field equations.

Example. Two interesting concrete examples of Lagrangians satisfying the hypotheses of Theorem 4 are:

- the Hilbert Lagrangian of general relativity, see [22, 8];

- the Lagrangian of Gauss-Bonnet gravity, see 8 .

In both these cases, it was proven in the cited papers that: $\lambda=\lambda_{V T}$, therefore, we will have:

$$
\Phi_{\lambda}=\Theta_{\lambda} .
$$

Yet, for general Lagrangians, the principal and the canonical Lepage equivalents will not coincide. Actually, the difference $\Phi_{\lambda}-\Theta_{\lambda}$ measures the failure of $\Theta_{\lambda}$ from having the closure property, as shown below.

Proposition 5 For a Lagrangian $\lambda=\lambda_{V T}+h d \alpha$ as in (37), there holds, up to the corresponding jet projections:

$$
\Phi_{\lambda}=\Theta_{\lambda}+\left(d \alpha-\Theta_{h d \alpha}\right) .
$$

Proof. From the linearity of $\Theta$, we have: $\Theta_{\lambda}=\Theta_{\lambda_{V T}}+\Theta_{h d \alpha}$. Adding to both hand sides $d \alpha$ and taking into account that, up to jet projections, $\Phi_{\lambda}=\Theta_{\lambda_{V T}}+d \alpha$, this leads to (41).

The term $d \alpha-\Theta_{h d \alpha}$ in (41) is 1-contact. Therefore, using (21), there exists a 1-contact form $\nu$ such that, up to the corresponding jet projections:

$$
d \alpha-\Theta_{h d \alpha}=p_{1} d \nu
$$

\subsection{First order Lagrangians}

For first order Lagrangians, the canonical Lepage equivalent will be of order $4 r-2=2$. In the following, for this particular case, we will determine the precise coordinate expression of $\nu$ in (42).

Proposition 6 For an arbitrary first order Lagrangian $\lambda \in \Omega_{n, X}\left(V^{1}\right)$, the following statements hold:

(i) $\lambda=\lambda_{V T}+\left(d_{i} \alpha^{i}\right) \omega_{0}$, where $\alpha^{i}=\alpha^{i}\left(x^{j}, y^{\sigma}, y^{\sigma}{ }_{j}\right)$ are of order 1 ;

(ii) the canonical and the principal Lepage equivalents of $\lambda$ are related by:

$$
\Phi_{\lambda}=\left(\pi^{2,1}\right)^{*} \Theta_{\lambda}+p_{1} d \nu
$$

where

$$
\nu:=\frac{1}{4}\left(\frac{\partial \alpha^{j}}{\partial y_{i}^{\sigma}}-\frac{\partial \alpha^{i}}{\partial y_{j}^{\sigma}}\right) \omega^{\sigma} \wedge \omega_{i j}
$$

Proof. (i) The form $\alpha=I \Theta_{\lambda}+\mu_{0}$ is of order 1 and $\pi^{1}$-horizontal, therefore it is locally expressed as $\alpha=\alpha^{i} \omega_{i}$, with $\alpha^{i}=\alpha^{i}\left(x^{j}, y^{\sigma}, y_{j}^{\sigma}\right)$ only (the precise expression of $\alpha^{i}$ can be found by applying (29) to $\Theta_{\lambda}$, but it is less essential in the following). The statement then follows from $h d \alpha=\left(d_{i} \alpha^{i}\right) \omega_{0}$. 
(ii) With $\alpha$ as above, we have: $\Phi_{\lambda}=\Theta_{\lambda_{V T}}+\left(\pi^{2,1}\right)^{*} d \alpha$. The Lagrangian $h d \alpha=: \mathcal{L}_{0} \omega_{0}$ (where $\left.\mathcal{L}_{0}=d_{k} \alpha^{k}\right)$ is of second order, therefore its principal Lepage equivalent is expressed as

$$
\Theta_{h d \alpha}=\mathcal{L}_{0} \omega_{0}+B^{i}{ }_{\sigma} \omega^{\sigma} \wedge \omega_{i}+B_{\sigma}^{i j} \omega^{\sigma}{ }_{j} \wedge \omega_{i},
$$

where $B_{\sigma}^{i}, B_{\sigma}^{i j}$ are given by (19). That is,

$$
B_{\sigma}^{i j}=\frac{\partial \mathcal{L}_{0}}{\partial y_{i j}^{\sigma}}=\frac{\partial}{\partial y_{i j}^{\sigma}}\left(d_{k} \alpha^{k}\right)=\frac{1}{2}\left(\frac{\partial \alpha^{i}}{\partial y_{j}^{\sigma}}+\frac{\partial \alpha^{j}}{\partial y_{i}^{\sigma}}\right)
$$

and from $\frac{\partial \mathcal{L}_{0}}{\partial y_{i}^{\sigma}}=\frac{\partial}{\partial y_{i}^{\sigma}}\left(d_{k} \alpha^{k}\right)=d_{k}\left(\frac{\partial \alpha^{k}}{\partial y_{i}^{\sigma}}\right)+\frac{\partial \alpha^{i}}{\partial y^{\sigma}}$, we find:

$$
B_{\sigma}^{i}=\frac{\partial \alpha^{i}}{\partial y^{\sigma}}+\frac{1}{2} d_{j}\left(\frac{\partial \alpha^{j}}{\partial y_{i}^{\sigma}}-\frac{\partial \alpha^{i}}{\partial y_{j}^{\sigma}}\right)
$$

Substituting into $\Theta_{h d \alpha}$ and taking into account that $\left(\pi^{2,1}\right)^{*} d \alpha=\left(d_{k} \alpha^{k}\right) \omega_{0}+\frac{\partial \alpha^{i}}{\partial y^{\sigma}} \omega^{\sigma} \wedge \omega_{i}+\frac{\partial \alpha^{i}}{\partial y^{\sigma}{ }_{j}} \omega_{j}^{\sigma} \wedge$ $\omega_{i}$, we finally get:

$$
\left(\pi^{2,1}\right)^{*} d \alpha-\Theta_{h d \alpha}=\frac{1}{2} d_{j}\left(\frac{\partial \alpha^{i}}{\partial y_{j}^{\sigma}}-\frac{\partial \alpha^{j}}{\partial y_{i}^{\sigma}}\right) \omega^{\sigma} \wedge \omega_{i}+\frac{1}{2}\left(\frac{\partial \alpha^{i}}{\partial y_{j}^{\sigma}}-\frac{\partial \alpha^{j}}{\partial y_{i}^{\sigma}}\right) \omega_{j}^{\sigma}{ }_{j} \wedge \omega_{i}
$$

On the other hand, a direct computation using: $d \omega^{\sigma}=-\omega^{\sigma}{ }_{k} \wedge d x^{k}$ and $d x^{k} \wedge \omega_{i j}=\delta^{k}{ }_{j} \omega_{i}-\delta_{i}^{k} \omega_{j}$ shows that $p_{1} d \nu$ is precisely the right hand side of the above. The statement then follows from (41).

\section{Reduced Lepage equivalents}

In the following, we present an alternative construction, which is advantageous in the case when the Lagrangian $\lambda$ can be order-reduced; for reducibility criteria, see, e.g., 6] [18, 19.

Consider a Lagrangian $\lambda \in \Omega_{n, X}\left(W^{r}\right)$, where $W^{r} \subset J^{r} Y$ is open, and pick any equivalent Lagrangian $\lambda^{\prime} \in \Omega_{n, X}\left(W^{s}\right)$ to $\lambda$, of minimal order $s \leq r$. Then, again, we can write

$$
\lambda=\left(\pi^{r, s}\right)^{*} \lambda^{\prime}+h d \alpha,
$$

for some $\alpha \in \Omega_{n-1}\left(W^{r-1}\right)$.

In particular, for a trivial Lagrangian $\lambda$, minimal order Lagrangians equivalent to $\lambda$ are $\pi^{r}$ projectable $n$-forms $\lambda^{\prime}=f\left(x^{i}\right) \omega_{0}$.

Proposition 7 Let $\lambda \in \Omega_{n, X}\left(W^{r}\right)$ be an arbitrary Lagrangian and $\lambda^{\prime} \in \Omega_{n, X}\left(W^{s}\right)$, a dynamically equivalent Lagrangian to $\lambda$, of minimal order $s \leq r$. Then:

(i) The $n$-form

$$
\phi_{\lambda}:=\Theta_{\lambda^{\prime}}+d \alpha
$$

where $\lambda^{\prime}$ and $\alpha$ are as in (47) and the equality must be understood up to the corresponding jet projections, is a Lepage equivalent of $\lambda$.

(ii) If $\lambda$ is variationally trivial, then any $\phi_{\lambda}$ constructed as above is closed. 
Proof. (i) The proof is similar to the one of Theorem 3 First, we note that, up to jet projections:

$$
h \phi_{\lambda}=h \Theta_{\lambda^{\prime}}+h d \alpha=\lambda^{\prime}+h d \alpha=\lambda
$$

moreover, $d \phi_{\lambda}=d \Theta_{\lambda^{\prime}}$ implies $p_{1} d \phi_{\lambda}=p_{1} d \Theta_{\lambda^{\prime}}=\mathcal{E}_{\lambda^{\prime}}=\mathcal{E}_{\lambda}$, which is a source form, that is, $\phi_{\lambda}$ is a Lepage equivalent of $\lambda$.

(ii) If $\lambda$ is trivial, then $\lambda^{\prime}=f\left(x^{i}\right) \omega_{0}$, which gives: $\Theta_{\lambda^{\prime}}=\lambda^{\prime}$. But, as $\lambda^{\prime}$ is an $n$-form on $X$, $\operatorname{dim} X=n$, we find that: $d \phi_{\lambda}=d \Theta_{\lambda^{\prime}}=d \lambda^{\prime}=0$.

Definition 8 We will call any Lepage equivalent built as in (47)-(48), a reduced Lepage equivalent of $\lambda$.

\section{Remarks.}

1. The reduced Lagrangian $\lambda^{\prime}$ of $\lambda$ (if it exists) is, generally, not unique. As a consequence, we may obtain multiple reduced Lepage equivalents $\phi_{\lambda}$ for the same Lagrangian. Even so, the multi-valued correspondence $\lambda \mapsto \phi_{\lambda}$ is $\mathbb{R}$-linear, in the following sense: for any $\lambda_{1}, \lambda_{2} \in$ $\Omega_{n, X}\left(W^{r}\right)$ and $a_{1}, a_{2} \in \mathbb{R}$, if $\phi_{\lambda_{1}} \in \phi\left(\lambda_{1}\right)$ and $\phi_{\lambda_{2}} \in \phi\left(\lambda_{2}\right)$, then $a_{1} \phi_{\lambda_{1}}+a_{2} \phi_{\lambda_{2}}$ belongs to the image $\phi\left(a_{1} \lambda_{1}+a_{2} \lambda_{2}\right)$.

2. The splitting (47) is, generally, only local - therefore, reduced Lepage equivalents are, in general, defined only locally.

In particular, for second order Lagrangians, we obtain:

Proposition 9 Any reducible second order Lagrangian admits a local first order Lepage equivalent.

Proof. If $\lambda \in \Omega_{n, X}\left(W^{2}\right)$ is reducible to a first order Lagrangian $\lambda^{\prime} \in \Omega_{n, X}\left(W^{1}\right)$, the corresponding reduced Lepage equivalent $\phi_{\lambda}$ is of order 1 , as both $\Theta_{\lambda^{\prime}}$ and $\alpha$ are, in this case, of order 1.

\section{Examples}

\subsection{Hilbert Lagrangian}

The Hilbert Lagrangian $\lambda_{g}$ is a peculiar example; in this case, we will see below that:

$$
\Phi_{\lambda_{g}}=\Theta_{\lambda_{g}}=\phi_{\lambda_{g}}
$$

where $\phi_{\lambda_{g}}$ corresponds to the famous non-invariant, first order Lagrangian equivalent to $\lambda_{g}$. In particular, $\Phi_{\lambda_{g}}$ is of order 1 .

The first equality above has already been discussed in Section 3. The second one is based on a result in [23], as follows. Denote by $Y=\operatorname{Met}(X)$, the bundle of nondegenerate tensors of type $(0,2)$ over $X$ and by $\left(g_{i j} ; g_{i j, k} ; g_{i j, k l}\right)$, the coordinates in a fibered chart on $J^{2} Y$. The Hilbert Lagrangian

$$
\lambda_{g}=\mathcal{R} \omega_{0}, \quad \mathcal{R}:=R \sqrt{|\operatorname{det} g|}
$$


can be split as $\lambda_{g}=\lambda_{g}^{\prime}+\left(d_{i} \alpha^{i}\right) \omega_{0}$, where

$$
\lambda_{g}^{\prime}=g^{j k}\left(\Gamma_{j l}^{i} \Gamma_{k i}^{l}-\Gamma_{j k}^{i} \Gamma_{i l}^{l}\right) \sqrt{|\operatorname{det} g|} \omega_{0}=: \mathcal{L}_{g}^{\prime} \omega_{0}
$$

is the reduced (non-invariant) Lagrangian for $\lambda_{g}$ and:

$$
\alpha=\left(\Gamma_{j}^{i j}-\Gamma_{j}^{j i}\right) \sqrt{|\operatorname{det} g|} \omega_{i} .
$$

In [23], Sec. 5.5.1, it was shown that:

$$
\Theta_{\lambda_{g}}=\Theta_{\lambda_{g}^{\prime}}+d \alpha
$$

which proves the second equality (49).

The coordinate expression of $\Theta_{\lambda_{g}}$ in the natural basis $\left\{d x^{i}, d g_{j k}, d g_{j k, i}\right\}$ of $\Omega\left(J^{1} Y\right)$ is known, [13], as:

$$
\begin{aligned}
\Theta_{\lambda_{g}}= & g^{i p}\left(\Gamma^{j}{ }_{i p} \Gamma^{k}{ }_{j k}-\Gamma_{i k}^{j} \Gamma^{k}{ }_{j p}\right) \sqrt{|\operatorname{det} g|} \omega_{0} \\
& +\left(g^{j p} g^{i q}-g^{p q} g^{i j}\right) \sqrt{|\operatorname{det} g|}\left(d g_{p q, j}+\Gamma_{p q}^{k} d g_{j k}\right) \wedge \omega_{i} .
\end{aligned}
$$

Let us explicitly calculate in the following the term $\Theta_{\lambda_{q}^{\prime}}$ in (51). This will only contain a $d g_{p q^{-}}$ component - or, in the contact basis, an $\omega_{(p q)}$-component, where $\omega_{(p q)}=d g_{p q}-g_{p q, r} d x^{r}$. More precisely,

$$
\Theta_{\lambda_{g}^{\prime}}=\mathcal{L}_{g}^{\prime} \omega_{0}+\frac{\partial \mathcal{L}_{g}^{\prime}}{\partial g_{p q, r}} \omega_{(p q)} \wedge \omega_{r}
$$

The derivative $\frac{\partial \mathcal{L}_{g}^{\prime}}{\partial g_{p q, r}}$ can be calculated directly, using $\mathcal{L}_{g}^{\prime}=g^{i h}\left(g^{j k} g^{l m}-g^{j l} g^{m k}\right) \Gamma_{h j l} \Gamma_{m k i}$ and $\frac{\partial \Gamma_{h j l}}{\partial g_{p q, r}}=\frac{1}{2}\left(\delta_{h}^{p} \delta_{j}^{q} \delta_{l}^{r}+\delta_{h}^{p} \delta_{l}^{q} \delta_{j}^{r}-\delta_{j}^{p} \delta_{l}^{q} \delta_{h}^{r}\right)$. We obtain:

$$
\frac{\partial \mathcal{L}_{g}^{\prime}}{\partial g_{p q, r}}=\Gamma^{r p q}-g^{q r} \Gamma_{k}^{k p}+\frac{1}{2} g^{p q}\left(\Gamma_{j}^{j r}-\Gamma_{j}^{r j}\right)
$$

\subsection{Klein-Gordon field Lagrangian}

A simplest and somwehat suprising example is provided by the classical Klein-Gordon field Lagrangian.

Consider the trivial bundle $Y=\mathbb{R}^{4} \times \mathbb{R}$, where $X=\mathbb{R}^{4}$ is equipped with the Minkowski metric $\eta=\operatorname{diag}(1,-1,-1,-1)$ (and corresponding Cartesian coordinates $\left.\left(x^{i}\right)_{i=\overline{1,4}}\right)$. We denote a set of global fibered coordinates on $Y$ by $\left(x^{i}, \varphi\right)$ and by $\left(x^{i}, \varphi, \varphi_{i}, \varphi_{i j}\right)$ the induced coordinates on $J^{2} Y$. The contact basis elements on $\Omega\left(J^{2} Y\right)$ will then be denoted as: $\tilde{\omega}=d \varphi-\varphi_{i} d x^{i}, \tilde{\omega}_{i}=d \varphi_{i}-\varphi_{i j} d x^{j}$.

The Klein-Gordon field Lagrangian $\lambda=\mathcal{L} \omega_{0} \in \Omega_{4}\left(J^{1} Y\right)$ is given (see, e.g., [4) by:

$$
\mathcal{L}=\frac{1}{2}\left(\eta^{i j} \varphi_{i} \varphi_{j}-m^{2} \varphi^{2}\right)
$$

where $m \geq 0$ is a constant. 
- Since the above Lagrangian is of first order (i.e., it is of minimal order in its equivalence class), its principal Lepage equivalent is also a minimal one. We immediately obtain:

$$
\phi_{\lambda}=\Theta_{\lambda}=\mathcal{L} \omega_{0}+\left(\eta^{i j} \varphi_{j}\right) \tilde{\omega} \wedge \omega_{i} .
$$

- As $\lambda$ is of order 1 , the canonical Lepage equivalent $\Phi_{\lambda}$ can be most easily determined from (43)-(44).

The unique Euler-Lagrange expression $\mathcal{E}:=\mathcal{E}_{\sigma}$ of $\lambda$ is: $\mathcal{E}=-\left(\eta^{i j} \varphi_{i j}+m^{2} \varphi\right)$, which allows us to calculate the associated Vainberg-Tonti Lagrangian $\lambda_{V T}=\mathcal{L}_{V T} \omega_{0}$ of $\lambda$ as:

$$
\mathcal{L}_{V T}=-\varphi \int_{0}^{1}\left(\eta^{i j} t \varphi_{i j}+m^{2} t \varphi\right) d t=-\frac{1}{2}\left(\eta^{i j} \varphi \varphi_{i j}+m^{2} \varphi^{2}\right) ;
$$

this gives: $\mathcal{L}=\mathcal{L}_{V T}+\left(d_{i} \alpha^{i}\right)$, with:

$$
\alpha^{i}=\frac{1}{2} \eta^{i j} \varphi \varphi_{j}
$$

Noticing that $\frac{\partial \alpha^{i}}{\partial \varphi_{j}}=\frac{\partial \alpha^{j}}{\partial \varphi_{i}}=\frac{1}{2} \eta^{i j} \varphi$, we find $\nu=\frac{1}{4}\left(\frac{\partial \alpha^{j}}{\partial \varphi_{i}}-\frac{\partial \alpha^{i}}{\partial \varphi_{j}}\right) \tilde{\omega} \wedge \omega_{i j}=0$, which means that $\Phi_{\lambda}=\Theta_{\lambda}$. All in all, we obtain,

$$
\Phi_{\lambda}=\Theta_{\lambda}=\varphi_{\lambda},
$$

though, in this case, $\lambda_{V T}$ and $\lambda$ do not coincide.

\subsection{Electromagnetic field Lagrangian}

Another standard example of a first order field Lagrangian is the electromagnetic field Lagrangian (e.g., [4). Consider the cotangent bundle $Y=T^{*} \mathbb{R}^{4}$ over the Minkowski spacetime $\left(\mathbb{R}^{4}, \eta\right)$. We denote the coordinates in a fibered chart on $J^{2} Y$ by $\left(x^{i} ; A_{i} ; A_{i, j} ; A_{i, j k}\right)$ and the contact basis 1 -forms by $\tilde{\omega}_{i}=d A_{i}-A_{i, j} d x^{j}, \tilde{\omega}_{i, j}=d A_{i, j}-A_{i, j k} d x^{k}$.

We will investigate the Lagrangian $\lambda=\mathcal{L} \omega_{0} \in \Omega_{4, X}\left(J^{1} Y\right)$, with:

$$
\mathcal{L}=F_{i j} F^{i j},
$$

where $F_{i j}=A_{j, i}-A_{i, j}$ and $F^{i j}=\eta^{i k} \eta^{j l} F_{k l}$.

- Noting that $\frac{\partial F_{i j}}{\partial A_{k, l}}=\delta_{j}^{k} \delta_{i}^{l}-\delta_{i}^{k} \delta_{j}^{l}$, we find:

$$
\frac{\partial \mathcal{L}}{\partial A_{j, i}}=4 F^{i j},
$$

which gives the principal Lepage equivalent:

$$
\Theta_{\lambda}=\mathcal{L} \omega_{0}+\frac{\partial \mathcal{L}}{\partial A_{j, i}} \tilde{\omega}_{j} \wedge \omega_{i}=\mathcal{L} \omega_{0}+4 F^{i j} \tilde{\omega}_{j} \wedge \omega_{i} .
$$

As the Lagrangian is a first order one, this is also a minimal Lepage equivalent. 
A nice feature of $\Theta_{\lambda}$ is that, under gauge transformations $A_{i} \mapsto A_{i}+\partial_{i} f$ (with $f=f\left(x^{k}\right)$ defined on the base manifold $\left.\mathbb{R}^{4}\right), \Theta_{\lambda}$ remains invariant. This follows as, on the one hand, the basis contact forms $\tilde{\omega}_{j}$ are easily seen to be invariant (and the same is true for $\tilde{\omega}_{j, k}$, though this is unessential for the moment) and on the other hand, the coefficients $F_{i j}$ are gauge invariant.

- The Euler-Lagrange expressions

$$
\mathcal{E}^{j}=\frac{\partial \mathcal{L}}{\partial A_{j}}-d_{i}\left(\frac{\partial \mathcal{L}}{\partial A_{j, i}}\right)=-4 d_{i} F^{i j}
$$

lead to the associated Vainberg-Tonti Lagrangian $\lambda_{V T}=\mathcal{L}_{V T} \omega_{0}$, as follows:

$$
\mathcal{L}_{V T}=-4 A_{j} \int_{0}^{1} d_{i} F^{i j} t d t=-2 A_{j} d_{i} F^{i j} .
$$

(where we took into account that $A^{i, j} \circ \chi_{t}=t A^{i, j}$ ). We thus obtain the desired divergence expression $d_{i} \alpha^{i}=\mathcal{L}-\mathcal{L}_{V T}$ as:

$$
d_{i} \alpha^{i}=F_{i j} F^{i j}+2 A_{j} d_{i} F^{i j}=2 A_{j, i} F^{i j}+2 A_{j} d_{i} F^{i j}=d_{i}\left(2 A_{j} F^{i j}\right),
$$

which allows us to identify:

$$
\alpha^{i}=2 A_{l} F^{i l} .
$$

We will calculate the canonical Lepage equivalent $\Phi_{\lambda}$ using (43)-(44); we find that $p_{1} d \nu$ is given similarly to (46):

$$
p_{1} d \nu=\frac{1}{2} d_{j}\left(\frac{\partial \alpha^{i}}{\partial A_{k, j}}-\frac{\partial \alpha^{j}}{\partial A_{k, i}}\right) \tilde{\omega}_{k} \wedge \omega_{i}+\frac{1}{2}\left(\frac{\partial \alpha^{i}}{\partial A_{k, j}}-\frac{\partial \alpha^{j}}{\partial A_{k, i}}\right) \tilde{\omega}_{k, j} \wedge \omega_{i}
$$

To this aim, we first note that $\frac{\partial A^{l, i}}{\partial A_{k, j}}=\eta^{l k} \eta^{i j}$, which leads after a brief computation to:

$$
\frac{1}{2}\left(\frac{\partial \alpha^{i}}{\partial A_{k, j}}-\frac{\partial \alpha^{j}}{\partial A_{k, i}}\right)=A^{i} \eta^{j k}-A^{j} \eta^{i k}
$$

and finally

$$
p_{1} d \nu=\left(A^{i, k}-A^{j}{ }_{j} \eta^{i k}\right) \tilde{\omega}_{k} \wedge \omega_{i}+\left(A^{i} \eta^{j k}-A^{j} \eta^{i k}\right) \tilde{\omega}_{k, j} \wedge \omega_{i}
$$

Thus, the canonical Lepage equivalent $\Phi_{\lambda}=\left(\pi^{2,1}\right)^{*} \Theta_{\lambda}+p_{1} d \nu$ is of first order:

$$
\Phi_{\lambda}=\mathcal{L} \omega_{0}+\left(4 F^{i k}+A^{i, k}-A^{j}{ }_{, j} \eta^{i k}\right) \tilde{\omega}_{k} \wedge \omega_{i}+\left(A^{i} \eta^{j k}-A^{j} \eta^{i k}\right) \tilde{\omega}_{k, j} \wedge \omega_{i}
$$

(as the second order term arising from the presence of $A_{k, j l}$ in $\tilde{\omega}_{k, j}=d A_{k, j}-A_{k, j l} d x^{l}$, actually vanishes).

Another interesting remark is the following. $\Phi_{\lambda}$ is not invariant under gauge transformations $A_{i} \mapsto A_{i}+\partial_{i} f$, hence, it has less symmetries than the Lagrangian $\lambda$ (in particular, this points out that $\Phi$ does not possess the mapping property $\left.J^{r} \alpha^{*} \Phi_{\lambda}=\Phi_{J^{r} \alpha^{*} \lambda}\right)$. Yet, in the exterior derivative

$$
d \Phi_{\lambda}=\mathcal{E}_{\lambda}+2\left(2 \eta^{j k} \eta^{i l}-\eta^{i j} \eta^{k l}-\eta^{i k} \eta^{j l}\right) \tilde{\omega}_{j, l} \wedge \tilde{\omega}_{k} \wedge \omega_{i}
$$

gauge invariance is restored. 


\section{Conclusion and outlook}

In the present paper, we have proposed two notions of local Lepage equivalent $\theta_{\lambda}$ for Lagrangians $\lambda$ of arbitrary order $r \geq 1$, possessing the so-called closure property: the given Lepage equivalent is a closed differential form if and only if the Lagrangian $\lambda$ is trivial. Both these notions are constructed as

$$
\theta_{\lambda}=\Theta_{\bar{\lambda}}+d \alpha,
$$

i.e., by adding an exact form $d \alpha$ to the principal (Poincaré-Cartan) form $\Theta_{\bar{\lambda}}$ of an appropriately chosen equivalent Lagrangian $\bar{\lambda}=\lambda-h d \alpha$; this guarantees that all equivalent Lagrangians to $\lambda$ will have the same $d \theta$ :

$$
d \theta_{\lambda}=d \Theta_{\bar{\lambda}} .
$$

In the above, we studied the following choices for $\bar{\lambda}$ :

1. The Vainberg-Tonti Lagrangian $\lambda_{V T}=I \mathcal{E}_{\lambda}$, sharing the same Euler-Lagrange form $\mathcal{E}_{\lambda}$ with $\lambda$; this Lagrangian is uniquely (and, in a sense, canonically) defined by the given Lagrangian $\lambda$; the obtained Lepage equivalent is 1-contact, yet, generally, of higher order than the principal Lepage equivalent $\Theta_{\lambda}$.

2. A Lagrangian of minimal order, equivalent to $\lambda$, which leads to a computationally simplest possible (though, generally not unique) Lepage equivalent for $\lambda$. With such a choice, the exterior derivative $d \phi_{\lambda}$ takes the simple possible form, i.e., it is always 2 -contact, $\omega^{\sigma}$-generated and of minimal order; such $d \phi_{\lambda}$ is called in [18, a minimal Lepage extension of the EulerLagrange form $\mathcal{E}_{\lambda}$. In particular, for reducible second order Lagrangians, our construction gives a recipe for obtaining a first order Lepage equivalent.

In both the above cases (and, actually, for any choice of $\lambda$ ), the obtained $d \theta_{\lambda}$ is at most 2contact - which is the minimal possible degree of contactness for the exterior derivative of a Lepage equivalent. Besides simplicity, our algorithm (53) has also the advantage of generality - as, at least in principle, it might also allow for other interesting choices of $\bar{\lambda}$, while still preserving the property (54).

Moreover, basing our construction on the principal (Poincaré-Cartan) Lepage equivalent as in (53) -(54) has promising features, e.g., for Hamiltonian field theory. It is known (see, e.g., [16]), that, under certain regularity conditions on the Lagrangian function $\mathcal{L}$, the Hamilton equation (25) for the principal Lepage equivalent $\Theta_{\lambda}$ (called the Hamilton-de-Donder equation) becomes, indeed, equivalent to the Euler-Lagrange equation of $\lambda$, which is what one would expect from a "correct" Hamilton equation. Also, in some particular cases when the Lagrangian $\lambda$ fails to satisfy the regularity condition, a "regularization" procedure is proposed in [16] by passing to an equivalent, regular Lagrangian $\bar{\lambda}$ and considering the Hamilton equation for $\Theta_{\bar{\lambda}}$ (which is, thus, equivalent to the Euler-Lagrange equation for $\lambda$ ) as the relevant Hamilton equation for $\lambda$; in this case, choosing a Lepage equivalent as in (53)-(54) guarantees that the obtained Hamilton equation for $\theta_{\lambda}$ is a correct one. A more detailed study of regularizable Lagrangians and of the resulting Hamilton theory, using the proposed Lepage equivalents, will be done in the near future.

Acknowledgment. We are extremely grateful to prof. Demeter Krupka for drawing our attention towards the closure property topic and for useful and extensive talks on it and also, to Bence Racskó for helpful discussions on reducible second order Lagrangians. 


\section{References}

[1] D. Betounes, Extension of the classical Cartan form, Physical Review D 29(4), 599-606 (1984).

[2] D. Betounes, Differential geometric aspects of the Cartan form: Symmetry theory, Journal of Mathematical Physics 28, 2347 (1987).

[3] M. Crampin, D.J. Saunders, The Hilbert-Carathéodory Form for Parametric Multiple Integral Problems in the Calculus of Variations, Acta Applicandae Mathematicae 76, 37-55 (2003).

[4] J. Franklin, Classical Field Theory, Cambridge University Press, 2017.

[5] G. Giachetta, L. Mangiarotti, G. Sardanashvily, Advanced Classical Field Theory, World Scientific, 2009.

[6] D. R. Grigore, On an order reduction theorem in the Lagrangian formalism, Nuovo Cim. B111, 1439-1447 (1996).

[7] D.R. Grigore, O.T. Popp, On the Lagrange-Souriau form in classical field theory, Mathematica Bohemica 123(1) 73-86 (1998).

[8] M. Hohmann, C. Pfeifer, N. Voicu, Canonical variational completion and 4 D Gauss-Bonnet gravity, European Physical Journal Plus 136, 180 (2021).

[9] D. Krupka, Introduction to global variational geometry, Atlantis Press, 2015.

[10] D. Krupka, Some Geometric Aspects of Variational Problems in Fibred Manifolds, Folia Fac. Sci. Nat. Univ. Purk. Brunensis, Physica 14, Brno (Czechoslovakia), 1973.

[11] D. Krupka, A map associated to the Lepagian forms on the calculus of variations in fibred manifolds, Czechoslovak Mathematical Journal, 27 (102), 114-118 (1977).

[12] D. Krupka, On the higher order Hamilton theory in fibered spaces, Proc. Conf. Diff. Geom. Appl., Nove Mesto na Morave, Czechoslovakia, Sept. 5-9, 1983 (J.E. Purkyne Univ., Brno) 167-184 (1984).

[13] D. Krupka, O. Krupkova, D. Saunders, The Cartan form and its generalizations in the calculus of variations, International Journal of Geometric Methods in Modern Physics 07(04), 631-654 (2010).

[14] D. Krupka, J. Musilova, Trivial Lagrangians in field theory, Differential Geometry and its Applications 9, 293-105 (1998).

[15] O. Krupkova, Hamiltonian field theory revisited: A geometric approach to regularity, Steps in Differential Geometry, Proc. of the Colloquium on Differential Geometry, 25-30 July, 2000, Debrecen, Hungary.

[16] O. Krupkova, D. Smetanova, On regularization of variational problems in first-order field theory, Rendiconti del Circolo Matematico di Palermo, Serie II, Suppl. 66, 133-145 (2001).

[17] M. Palese, O. Rossi, F. Zanello, Geometric integration by parts and Lepage equivalents, arXiv:2010.16135 [math-ph] (2020). 
[18] O. Rossi, The Lagrangian Order-Reduction Theorem in Field Theories, Commun. Math. Phys. $362,107-128$ (2018).

[19] D. Saunders, On Lagrangians with Reduced-Order Euler-Lagrange Equations, Symmetry, Integrability and Geometry: Methods and Applications 14, 089 (2018).

[20] D.J. Saunders, M. Crampin, The fundamental form of a homogeneous Lagrangian in two independent variables, Journal of Geometry and Physics 60(11), 1681-1697 (2010).

[21] Z. Urban, J. Brajercik, The fundamental Lepage form in variational theory for submanifolds, Int. J. Geom. Methods in Modern Physics 15(6) 1850103 (2018).

[22] N. Voicu, D. Krupka, Canonical variational completion of differential equations, Journal of Mathematical Physics 56, 043507 (2015).

[23] N. Voicu, Source Forms and Their Variational Completions, in vol. The Inverse Problem of the Calculus of Variations - Local and Global Theory, ed. Dmitri Zenkov, Atlantis Press-Springer (2015). 